\title{
DYNAMIC THREE-DIMENSIONAL RECONNECTION IN A SEPARATOR GEOMETRY WITH TWO NULL POINTS
}

\author{
D. I. Pontin ${ }^{1}$ and I. J. D. CRaig \\ Department of Mathematics, University of Waikato, Private Bag 3105, Hamilton, New Zealand \\ Received 2005 August 22; accepted 2005 December 23
}

\begin{abstract}
The dynamic behavior of disturbances in the vicinity of a pair of magnetically connected three-dimensional null points is examined. The aim is to investigate how nonlinear disturbances lead to strong localized currents that initiate magnetic reconnection at the separator. The problem is formulated in an incompressible cylindrical geometry by superposing arbitrary disturbance fields onto a "background" two-null field. Two different regimes are found for the dynamic evolution, depending on the relative strengths of the background magnetic and velocity fields. In one regime, disturbance pulses split into ingoing and outgoing components, which propagate along the background field lines. In the other "flux pileup" regime, a strong driving flow localizes the disturbances toward the null point pair. Current structures aligned with the spines, fans, and separator present in the field are found to result, and the structure of these currents and their scaling with resistivity is investigated.
\end{abstract}

Subject headings: MHD — plasmas — Sun: flares — Sun: magnetic fields

Online material: color figures

\section{INTRODUCTION}

Magnetic reconnection is a process that is fundamental to many phenomena in astrophysical and laboratory plasmas. Reconnection is the only mechanism capable of releasing "topologically bound" magnetic energy, in the form of ohmic heating and the kinetic energy of mass motion. Most astrophysical plasmas, however, are highly conducting - the dimensionless collisional resistivity is an inverse Lundquist number of order $\eta \simeq 10^{-10}$ - and for reconnection to be effective, localized regions comprising huge magnetic field gradients, and therefore strong currents, must be present. In this paper, we investigate the growth of such intense currents in a magnetic separator topology.

We concentrate here on magnetic reconnection in the presence of three-dimensional null points of the magnetic field. The resulting "spine and fan" separatrix topology of the background field is outlined in $\S 2$. For the present, we emphasize that reconnection at separatrix surfaces, as well as at separator lines linking the nulls, is thought to be important in both solar and stellar atmospheres, as well as closer to home in the Earth's magnetosphere. In the solar corona in particular, it is predicted that there should be present an abundance of three-dimensional null points (e.g., Inverarity \& Priest 1999; Albright 1999; Longcope et al. 2003) and separators (Schrijver \& Title 2002; Beveridge \& Longcope 2005). It is further predicted that such sites should provide privileged regions for heating the corona (Longcope 1996; Antiochos et al. 2002; Priest et al. 2005). There is also observational evidence that reconnection at a three-dimensional null point may act as a trigger for some solar flares (Fletcher et al. 2001).

Our present aim is to study separator reconnection. Despite being invoked as an explanation for many dynamic phenomena in the corona, such as X-ray bright points (Longcope et al. 2001) and solar flares (Longcope \& Noonan 2000), the mechanisms of separator reconnection are not well understood. What is known is that separator reconnection occurs quite naturally on the Sun,

\footnotetext{
${ }^{1}$ Current address: Space Science Center, University of New Hampshire, Durham, NH 03824.
}

for example, when two photospheric flux sources move relative to one another in the presence of an overlying field (Parnell \& Galsgaard 2004). Observational evidence of separator reconnection in the corona has recently been presented by Longcope et al. (2005). In addition, it has been shown that flare statistics can be modeled using a superposition of separators (Wheatland 2002).

In this paper, we develop solutions that model current growth and reconnection at a separator. Due to the extremely low value of the resistivity in astrophysical plasmas, a crucial property of any reconnection model is the predicted reconnection rate, and whether it is sufficient to explain observed dynamic phenomena at realistic plasma parameters. To this end, we discuss the scaling properties of the peak current with resistivity in each of the regimes described.

The model is based on the principles first developed by Craig \& Henton (1995), who proposed a steady state, resistive, planar model for reconnection at a two-dimensional null point. This work has since been generalized into three dimensions, first with the addition of an axial field to the two-dimensional X-point, such that there is no null in the field (Craig et al. 1995), and second to spine and fan reconnection at single three-dimensional nulls (Craig \& Fabling 1996). Further generalizations are possible to include the effects of time dependence (Craig \& Fabling 1998), as well as additional (nonresistive) nonideal effects (e.g., Ji \& Song 2001; Craig \& Watson 2003).

In what follows we develop a resistive, time-dependent model for reconnection occurring in a magnetic field in cylindrical geometry, where either one or two three-dimensional null points are present. This extends and generalizes the work of Watson \& Craig (2002), who made a preliminary investigation of similar configurations in a steady state regime (see also Tassi et al. 2003), and it provides the possibility of reconnection at curved current sheets and also separator current sheets. Although we consider here only resistive nonideal effects, we do not expect that the introduction of further nonideal effects would alter the qualitative results significantly. Hall currents, for example, are capable of having a profound effect on the detailed microphysics of the reconnection 
region. Their effect depends, however, both on the symmetries of the reconnection problem and on the presence of background "guide" fields (Craig \& Watson 2003, 2005). For the present, we simplify our discussion by concentrating on the buildup of strong field gradients in the vicinity of the reconnection region.

In $\S 2$ we discuss the topology of the magnetic fields considered. In $\S 3$ we introduce the governing MHD equations and describe the method of analysis, emphasizing in particular the wave properties of the solution, which we believe provide generic signatures for all transient magnetic merging solutions. In $\S \S 4$ and 5 the results of numerical simulations of the equations are discussed and their scalings with resistivity are summarized. We present our conclusions in $\S 6$.

\section{TOPOLOGY OF CONNECTED NULL POINT PAIRS}

We begin by summarizing the magnetic field topology associated with three-dimensional nulls. The field lines that asymptotically approach three-dimensional null points provide a local "skeleton" of the magnetic field. In the case of a single threedimensional null the skeleton comprises a "spine" line and a "fan" surface. The spine is a pair of field lines that approach (recede from) the null in opposite directions, while a family of field lines radiate out from (into) the null in the fan plane (e.g., Fukao et al. 1975; Lau \& Finn 1990; Parnell et al. 1996). By considering a Taylor expansion of $\boldsymbol{B}$ about the null point, it can be seen that the orientation of the spine and fan are determined by the eigenvectors of the matrix $\nabla \boldsymbol{B}$. This matrix is traceless, and the eigenvectors corresponding to the eigenvalues of like sign (or whose real parts have like sign) determine the orientation of the fan, while the other defines the spine. In general, the field strength in the fan will not be isotropic, and this anisotropy is determined by the fan eigenvalues.

When two null points are present a "separator" - a field line that directly connects the nulls - may exist. Kinematic considerations for fields containing multiple nulls (Lau \& Finn 1990; Priest \& Titov 1996) suggest that spines, fans, and separators provide special sites at which magnetic reconnection can occur, and this view has been well supported by steady state reconnection solutions (Craig et al. 1999).

In what follows we consider a two-null field whose separator is formed by the intersecting fan planes of the component nulls. This structure provides a generic separator geometry in the sense that the separator line is topologically stable, being robust to arbitrary, small perturbations in the background field. Note that although a separator could be formed by the coexistence of two null spines or one spine and one fan field line, neither of these structures is topologically stable, due to the uniqueness of the spine lines. This is one advantage of the present study over that of Craig et al. (1999), who consider a general class of fields containing multiple nulls in a steady state regime. In this case spinefan separators are present, which can only be maintained by the high degree of symmetry present in the field. We believe the geometry of the present study also provides a significant improvement on many previous studies of separator reconnection, which model the separator simply using a planar X-point threaded by an arbitrary axial field (e.g., Heerikhuisen \& Craig 2004). As we show below, the influence of the nulls themselves cannot be discounted when considering separator reconnection.

\section{THE MHD EQUATIONS}

\subsection{Form of the Solution}

We assume the equations of collisional resistive MHD, based on an incompressible plasma in an open (unbounded) geom- etry. The problem is scaled according to the reference coronal values

$$
\begin{gathered}
B_{c}=10^{2} \mathrm{G}, \quad l_{c}=10^{9.5} \mathrm{~cm}, \\
n_{c}=10^{9} \mathrm{~cm}^{-3}, \quad v_{\mathrm{A}}=10^{9} \mathrm{~cm} \mathrm{~s}^{-1}
\end{gathered}
$$

Time is now measured in units of the Alfvén time $\tau_{\mathrm{A}}=l_{c} / v_{\mathrm{A}}$, which is typically a few seconds in coronal applications. In this formulation the plasma resistivity is an inverse Lundquist number $\eta \simeq 10^{-14}$. The simplifying assumption is made that the viscosity $\nu$ is isotropic. In the simulations described below, $\nu$ is chosen to scale linearly with $\eta$ - this choice has been shown to reproduce the pure resistive scalings of the current layer (Craig \& Watson 2005).

The dimensionless induction equation is given by

$$
\frac{\partial \boldsymbol{B}}{\partial t}=\nabla \times(\boldsymbol{V} \times \boldsymbol{B})+\eta \nabla^{2} \boldsymbol{B}
$$

Taking the curl of the viscous momentum equation, we obtain

$$
\frac{\partial \Omega}{\partial t}=\nabla \times(\boldsymbol{J} \times \boldsymbol{B})-\nabla \times(\Omega \times \boldsymbol{V})+\nu \nabla^{2} \Omega,
$$

where $\boldsymbol{J}=\nabla \times \boldsymbol{B}$ is the current, $\Omega=\nabla \times \boldsymbol{V}$ is the vorticity, and $\eta$ and $\nu$ are assumed constant. We must also impose the constraint equations

$$
\nabla \cdot \boldsymbol{B}=0, \quad \nabla \cdot \boldsymbol{V}=0
$$

Once the magnetic and velocity fields have been found, the plasma pressure may be obtained from the "uncurled" form of the momentum equation. The assumption of an incompressible plasma $(\nabla \cdot V=0)$ is made in order to facilitate our method of solution, since in this form the equations display a high degree of symmetry between $\boldsymbol{B}$ and $\boldsymbol{V}$ (Craig \& Henton 1995). Furthermore, once the current sheet has formed, its behavior is expected to be largely incompressible, as the global timescale for merging is typically much longer than the timescale for fast mode propagation across the current sheet. It has been shown that finite compressibility has only a weak effect on the scaling properties of flux pileup current sheets and in fact acts to marginally speed up the resultant reconnection (see Litvinenko \& Craig 2003 and references therein).

In order to construct reconnection solutions, we use the superposition technique of Craig \& Henton (1995) and let

$$
\boldsymbol{B}=\beta \boldsymbol{P}(\boldsymbol{x})+\boldsymbol{b}(\boldsymbol{x}, t), \quad \boldsymbol{V}=\alpha \boldsymbol{P}(\boldsymbol{x})+\boldsymbol{v}(\boldsymbol{x}, t),
$$

where $\boldsymbol{P}$ is a steady state potential "background" field and $\boldsymbol{b}$ and $\boldsymbol{v}$ are "disturbance" fields of arbitrary amplitude. The method of solution is based on choosing a form of reduced dimensionality for the disturbance fields such that equations (2) and (3) may be reduced to a system of ordinary differential equations. In the steady state regime, this corresponds to automatically satisfying the momentum equation, leaving only the induction equation to be solved. In this case, $\boldsymbol{v}(\boldsymbol{x})=(\beta / \alpha) \boldsymbol{b}(\boldsymbol{x})$, and in order for the momentum equation to be automatically satisfied we require

$$
\nabla \times[(\nabla \times \boldsymbol{b}) \times \boldsymbol{b}]=0
$$


In previous models based on Cartesian geometry, $\boldsymbol{b}$ has taken either the form $\boldsymbol{b}=f(x, y) \hat{z}$ or $\boldsymbol{b}=f(x) \hat{\boldsymbol{y}}+g(x) \hat{z}$ (or cyclic permutations thereof $)$. It is interesting to note that these forms are in fact overrestrictive for this method, in that they satisfy $(\nabla \times b) \times$ $\boldsymbol{b}=0$, whereas this quantity need only be curl-free.

In cylindrical coordinates we find that there is a reduced choice of such low-dimensionality disturbances. Of those forms that satisfy the divergence condition, the only two that additionally satisfy equation (6) are

$$
\boldsymbol{b}_{1}=b(r, \theta, t) \hat{z}, \quad \boldsymbol{b}_{2}=a(r, t) \hat{\boldsymbol{\theta}}+b(r, t) \hat{z} .
$$

In contrast to the Cartesian case, these forms do not satisfy the more restrictive condition $(\nabla \times \boldsymbol{b}) \times \boldsymbol{b}=0$. The disturbance form of most interest to us is $\boldsymbol{b}_{1}$, since the second form describes disturbance field structures that vary only in the radial direction. Disturbances based on $\boldsymbol{b}_{1}$ provide a richer structure to the resulting currents, and can in any case be used to model a single component of the form $\boldsymbol{b}_{2}$ by "turning off" the $\theta$-dependence.

Guided by the above considerations, we take

$$
\boldsymbol{B}=\beta \boldsymbol{P}+b(r, \theta, t) \hat{\boldsymbol{z}}, \quad \boldsymbol{V}=\alpha \boldsymbol{P}+v(r, \theta, t) \hat{\boldsymbol{z}} .
$$

By analogy with the Cartesian case, we look for a potential field $\boldsymbol{P}=\left[P_{1}(r), P_{2}(r, \theta), P_{3}(r, z)\right]$ that is linear in $\theta$ and $z$. The only potential field of this form is

$$
\boldsymbol{P}=\left\{-\left[\frac{\gamma \ln (r / l)}{(r / l)}+\frac{\kappa r}{2 l}\right], \frac{\gamma \theta}{(r / l)}, \frac{\kappa z}{l}\right\}
$$

where $\gamma, \kappa$, and $l$ are constants. We note that the field is singular at the origin, and nonperiodic in $\theta$, and so the solution may not be considered as global, but rather must be considered valid only in some restricted annular domain that excludes the origin and the negative $x$-axis $(\theta= \pm \pi)$. The background field $\boldsymbol{P}$ may contain one or two null points, depending on the values of $\gamma$ and $\kappa$, while $l$ is a scale factor that determines the distance of the null point pair from the origin. If $\gamma$ and $\kappa$ are of the same sign, then only a single null is present; however, if $\gamma$ and $\kappa$ are of opposite sign and $|\gamma|>|\kappa| e$, then two nulls are present.

The skeleton of the field in each case is shown in Figure 1. Here we concentrate primarily on the case in which two nulls are present in the field. As shown in the figure, the nulls are joined by a separator (along $\theta=z=0$ ), formed by the intersection of their fan planes.

\subsection{Equations for the Disturbance Fields}

Substituting the above expressions for $\boldsymbol{B}$ and $\boldsymbol{V}$ (eqs. [8] and [9]) into equations (2) and (3), we find that

$$
\begin{gathered}
b_{t}=\alpha\left(\frac{\kappa}{l}-\mathcal{L}\right) b-\beta\left(\frac{\kappa}{l}-\mathcal{L}\right) v+\eta \nabla^{2} b \\
v_{t}=-\alpha\left(\frac{\kappa}{l}+\mathcal{L}\right) v+\beta\left(\frac{\kappa}{l}+\mathcal{L}\right) b+\nu \nabla^{2} v+g(t),
\end{gathered}
$$

where

$\mathcal{L}=P_{1} \partial_{r}+\frac{P_{2}}{r} \partial_{\theta}=-\left[\frac{\gamma l \ln (r / l)}{r}+\frac{\kappa r}{2 l}\right] \partial_{r}+\frac{\gamma l \theta}{r^{2}} \partial_{\theta}$

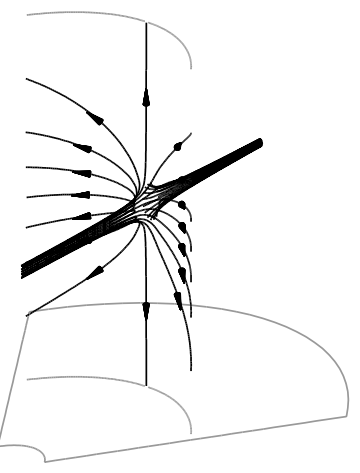

(a)

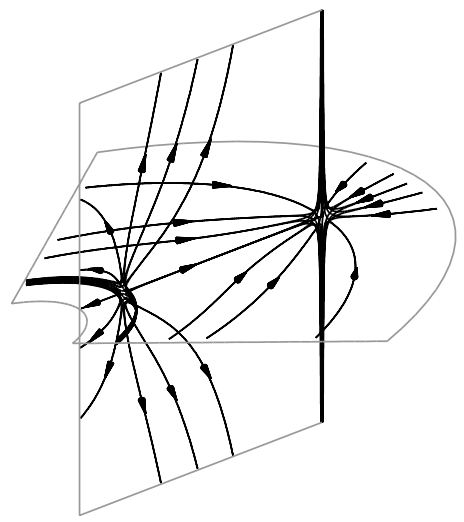

(b)
Fig. 1.-Field lines of the skeleton of the background field $\boldsymbol{P}$, when $(a) \kappa$ and $\gamma$ are of the same sign and a single null is present, and $(b) \kappa$ and $\gamma$ are opposite signs and $|\gamma|>|\kappa| e$, giving two nulls (with $\kappa>0$ in each case). The gray lines indicate the shape of the domain and the locations of the fan planes of the nulls.

is the directional derivative along $\boldsymbol{P}$ in the $r-\theta$ plane,

$$
\nabla^{2} a=(1 / r)\left(r a_{r}\right)_{r}+\left(1 / r^{2}\right) a_{\theta \theta},
$$

and where subscripted letters denote partial derivatives. Equation (11) is obtained by integrating either component of equation (3) employing integration by parts, with the arbitrary function of time $g(t)$ being the constant of integration. Note that $g$ simply gives a time-varying but spatially uniform $z$-component of $\boldsymbol{V}$, that is, a uniform "shift" of the flow structure in the $z$-direction. Thus, in order to maintain the cospatial nature of the null points of the background magnetic and flow fields, we hereafter set $g=0$. A procedure analogous to that described above can be used to derive the corresponding equations when the disturbance field takes the form $\boldsymbol{b}_{2}$ in equation (7), as described in the Appendix.

\subsection{Wave Properties of the Solution}

In the majority of astrophysical plasmas, nonideal effects only become important when very small length scales develop, due to the fact that the resistive and viscous coefficients $(\eta$ and $\nu)$ are so small. It is therefore natural to examine the properties of our system in the ideal limit, when $\eta=\nu=0$. This problem is simplified by invoking the Elsasser variables $M=b-v$ and $N=$ $b+v$, which from equations (10) and (11) satisfy

$$
\begin{aligned}
M_{t} & =(\alpha-\beta) \frac{\kappa}{l} N-(\alpha+\beta) \mathcal{L} M, \\
N_{t} & =(\alpha+\beta) \frac{\kappa}{l} M-(\alpha-\beta) \mathcal{L} N .
\end{aligned}
$$

To simplify further, observe that the operator $\mathcal{L}$ must be expressible as a total derivative, since it defines the directional derivative along the background planar field. More formally, we can change from the $(r, \theta)$ coordinates to a system $(\psi, \chi)$ based on the (planar projected) field lines

$$
\frac{d r}{P_{1}}=\frac{r d \theta}{P_{2}}
$$

which are labeled by $\psi$. It follows that $\chi$ is a coordinate running along the field lines, such that $\nabla \psi \cdot \nabla \chi \neq 0$. By a suitable choice we can make the directional derivative $\mathcal{L} \rightarrow \partial_{\chi}$. The further 
change to a comoving frame $\tau=t, s=\chi-\alpha t$ reduces equations (14) and (15) to

$$
\begin{aligned}
& M_{\tau}=(\alpha-\beta) \frac{\kappa}{l} N-\beta M_{s}, \\
& N_{\tau}=(\alpha+\beta) \frac{\kappa}{l} M+\beta N_{s} .
\end{aligned}
$$

It follows from this that both $M$ and $N$, and thus $b$ and $v$, satisfy the generalized Klein-Gordon equation

$$
b_{\tau \tau}=\beta^{2} b_{s s}+\frac{\kappa^{2}}{l^{2}}\left(\alpha^{2}-\beta^{2}\right) b .
$$

This equation for the disturbance field highlights the wavelike nature of the problem.

First note that equation (19) has two characteristics,

$$
C^{ \pm}=s \pm \beta \tau=\chi-(\alpha \mp \beta) \tau \text {. }
$$

Obviously, if $|\alpha|>|\beta|$, then both characteristics correspond to waves propagating along the field lines in the positive $\chi$ direction, of different speeds. By contrast, if $|\alpha|<|\beta|$, then the characteristics correspond to two waves that propagate in opposite directions (see also Craig \& Fabling 1998). Thus, there are two different possible regimes for the solution, depending on the relative sizes of $\alpha$ and $\beta$. The waves are Alfvén waves, which represent the incompressible limit of (compressive) fast-mode Alfvénic disturbances.

A further key property is the possibility of growth in the solution. Obviously, growth, as opposed to oscillatory behavior, can occur only if the source term of equation (19) is positive, that is, $\alpha^{2}>\beta^{2}$. Note that equation (19) is a generalization of the Cartesian "fan" equation analyzed by Craig \& Fabling (1998) using Fourier transform methods. Applying their analysis to equation (19) suggests that the condition for growth derived above may be sufficient, even when small dissipation coefficients are accounted for. In any case, growth depends not only on a sufficiently strong driving flow, but also on the sign of the parameter $\kappa$ of the background field. In order to obtain growth, we require in addition that the background flow has the capacity to stretch and amplify the disturbance field, $b \hat{z}$. For this reason, we concentrate on the case $\kappa>0$.

\subsection{Numerical Simulations}

We now consider numerical simulations of equations (10) and (11) based on a purpose-built, predictor-corrector scheme on a cylindrical mesh. In all cases the potential background fields are perturbed by imposing a single initial magnetic field pulse $(b)$ at some location within the numerical domain. We first concentrate on two-null oscillatory $|\beta|>|\alpha|$ solutions, before going on to discuss flux pileup models. In all of the simulations we take $\nu=\eta$, since a linear scaling of $\nu$ with $\eta$ is known to preserve the purely resistive scalings (Craig \& Watson 2005). Note also that we find that the chosen value of $\nu$ has little effect on the results discussed.

\section{WAVELIKE REGIME $(|\beta|>|\alpha|)$}

According to the above analysis, when the background magnetic field dominates the driving flow the evolution of the disturbance field is characterized by inward and outward propagating waves. Thus, the initial disturbance is expected to spread along the background field lines, within an envelope bounded by peaks traveling in and moving out at speeds $\alpha \pm \beta$, as determined by equation (20). Figures 2, 3, and 4 show that this behavior is reproduced in the simulations. ${ }^{2}$ We see that the magnetic disturbance develops two separate peaks, which travel along the field lines of $\boldsymbol{P}$ toward and away from the null point pair. However, the geometry of the background field lines, and the location of the initial disturbance with respect to the background field structure, are found to influence, quite strongly, the localization of the ingoing pulse. In the present simulations the direction of the magnetic field and plasma flow are quite arbitrary: the flow may be in either direction, and although this influences the relative speeds of the peaks of the disturbance, the localization and current growth properties remain unaffected.

The background field structure is the two-null structure of Figure $1 b$. There is one null, hereafter named "null 1," whose fan lies in the $r-z$ plane and whose curved spine lies in the $r-\theta$ plane; and another (null 2) whose fan lies in the $r$ - $\theta$ plane and whose spine lies in the $z$-direction. The spine of each null bounds the fan surface of the other. If the initial pulse is located to the negative- $x$ side of the spine of null 1 , or if it disturbs this spine, then the disturbance spreads out and localizes to the fan of null 1, as shown Figure 2. However, if the disturbance is initiated in the fan of null 2 (i.e., to the positive- $x$ side of the spine of null 1 ), then in general the ingoing pulse is squeezed in toward null 2 along $\boldsymbol{P}$, where it localizes toward the spine, as in Figure 3.

In addition, a third type of behavior may occur. If the disturbance is initiated in the fan of null 2 , but sufficiently close to the spine of null 1, then the ingoing pulse will be transported inward toward the separator joining the two nulls, as in Figure 4, the disturbance being aligned with the fan plane of null 1 . The relative size of the region of space in which this separator localization occurs depends on the geometry of the field lines in the fan of null 2, or equivalently the isotropy of this null, since the disturbances propagate along $\boldsymbol{P}$. In each of the three cases described above, the outgoing peak moves outward along $\boldsymbol{P}$, spreading due to diffusion and the increasing plasma velocity away from the nulls.

Of central interest in each of the above cases is whether a growth in the current density occurs, and to what extent this depends on the resistivity $\eta$. As expected, the magnitude of both disturbance peaks decays in time, as there is no "flux pileup" occurring. The current associated with the outgoing pulse does indeed decay, but the ingoing pulse is always associated with current growth. The growth is stalled when resistive effects begin to dominate. Independent results for the advection of magnetic pulses suggest that in the absence of flux pileup, the peak current is controlled by the width $(\eta)^{1 / 2}$ of the current layer (e.g., Craig $\&$ Fabling 1998). This corresponds to a slow Sweet-Parker dissipation rate. We find here that while this scaling is not adhered to strictly in the present more complex field structure, it is approximately followed.

Figure 5 shows a logarithmic plot of peak current versus resistivity for each of the spine, fan, and separator cases. Thus, if $J_{\max }$ follows a power-law dependence of the form

$$
J_{\max } \sim \eta^{-\mu_{J}}
$$

then the gradient determines $\mu_{J}$. Also plotted, for comparison, are the results for a similar run in which a localization toward the curved fan plane of the single null field shown in Figure $1 a$ was

\footnotetext{
2 These figures show frames from animations, which can be viewed at http:// www.math.waikato.ac.nz/ math0097/cyl_anim.html.
} 

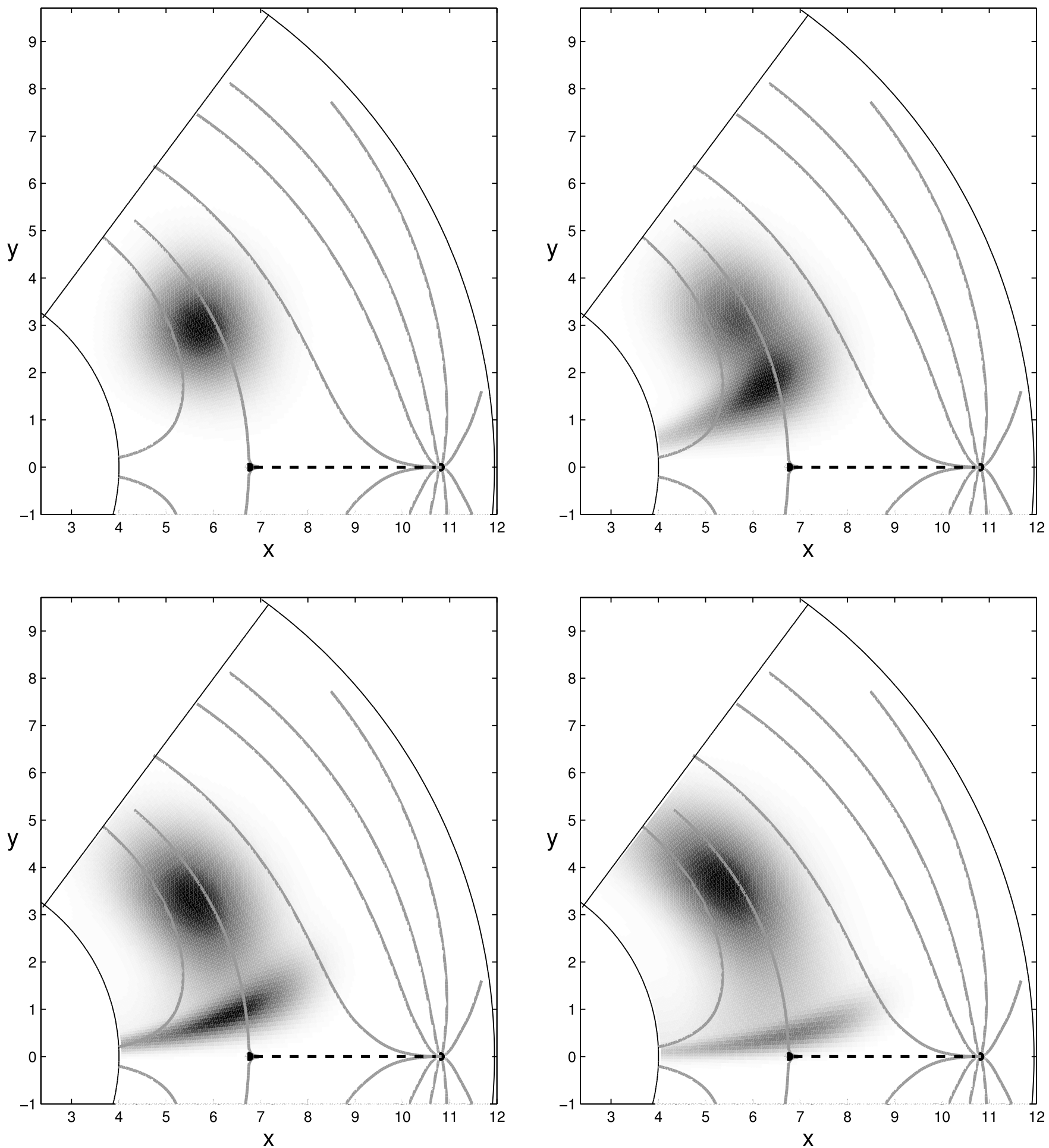

FIG. 2.-Evolution of a magnetic field disturbance ( gray scale) in $r \theta$, with the ingoing peak forming a current in the fan plane of null 1. The dots indicate the positions of the nulls, and the dotted line is the separator, while the gray lines show some planar-projected representative field lines of $\boldsymbol{P}$. The shading in each image, taken at times $t=0,3.2,6.3,11.0$, is scaled to the maximum in that frame, and the chosen parameters are $\alpha=0.8, \beta=1, \gamma=-1, \kappa=0.33$, and $l=5$. [See the electronic edition of the Journal for a color version of this figure.]

considered. The results are displayed in the top portion of Table 1. Note that while the fan current scaling is similar in the two cases considered, the spine and separator currents scale somewhat more slowly. These discrepancies can perhaps be attributed to the lack of highly localized current structures in the present cylindrical geometry. In any case, since the scaling of the peak current with resistivity is such that the resulting reconnection rate is unlikely to be energetically significant at realistic values of the resistivity, it seems more profitable to consider possible enhancements in the flux pileup regime with $|\alpha|>|\beta|$.

\section{FLUX PILEUP REGIME $(|\alpha|>|\beta|)$}

\subsection{Localization Phase}

We now examine the evolution of our system in the regime $|\alpha|>|\beta|$. Consider first the ideal limit with $\eta=\nu=0$. Since we 

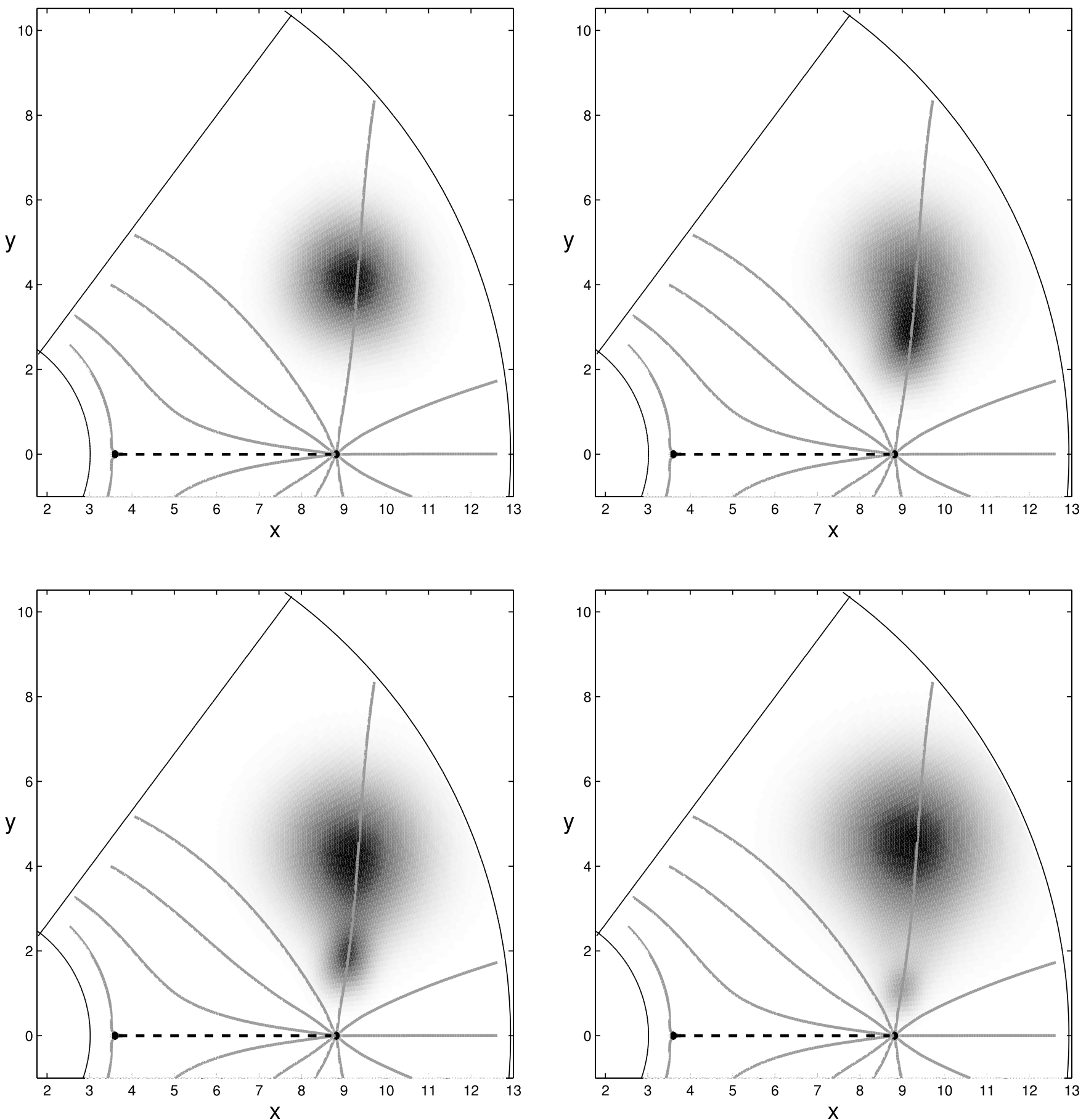

FIG. 3.- Symbols are the same as Fig. 2. The ingoing peak forms a current moving toward the spine of null 2. The shading in each image, taken at times $t=0,7.7,14.2$, 21.7 , is scaled to the maximum in that frame, and the chosen parameters are $\alpha=0.8, \beta=1, \gamma=-1, \kappa=0.25$, and $l=3$. [See the electronic edition of the Journal for a color version of this figure.]

require that our dynamic models must be able to reproduce the steady state solutions, in which $v=(\beta / \alpha) b$, we introduce the function $f=v-(\beta / \alpha) b$ to measure departures from the steady state. Now, rewriting equations (10) and (11) in terms of $b$ and $f$, we have in the ideal limit $(\eta=\nu=0)$ that

$$
\begin{gathered}
b_{t}+\alpha^{-} \mathcal{L} b=\frac{\kappa}{l} \alpha^{-} b-\beta\left(\frac{\kappa}{l}-\mathcal{L}\right) f, \\
f_{t}+\alpha^{+} \mathcal{L} f=-\alpha^{-} \frac{\kappa}{l} f-\frac{\beta}{\alpha} \alpha^{-}\left(\frac{\kappa}{l}-\mathcal{L}\right) b,
\end{gathered}
$$

where

$$
\alpha^{+}=\frac{\alpha^{2}+\beta^{2}}{\alpha}, \quad \alpha^{-}=\frac{\alpha^{2}-\beta^{2}}{\alpha} .
$$

The advection terms on the left-hand side of the above equations suggest that the field $f$ evolves more quickly than $b$, as the coefficients $\alpha^{+}$and $\alpha^{-}$determine inverse timescales for these processes, and $\alpha^{+}>\alpha^{-}$. If we now consider the limit of very strong flow, that is, $\beta / \alpha \ll 1$, and use the variable $s$ to compute 

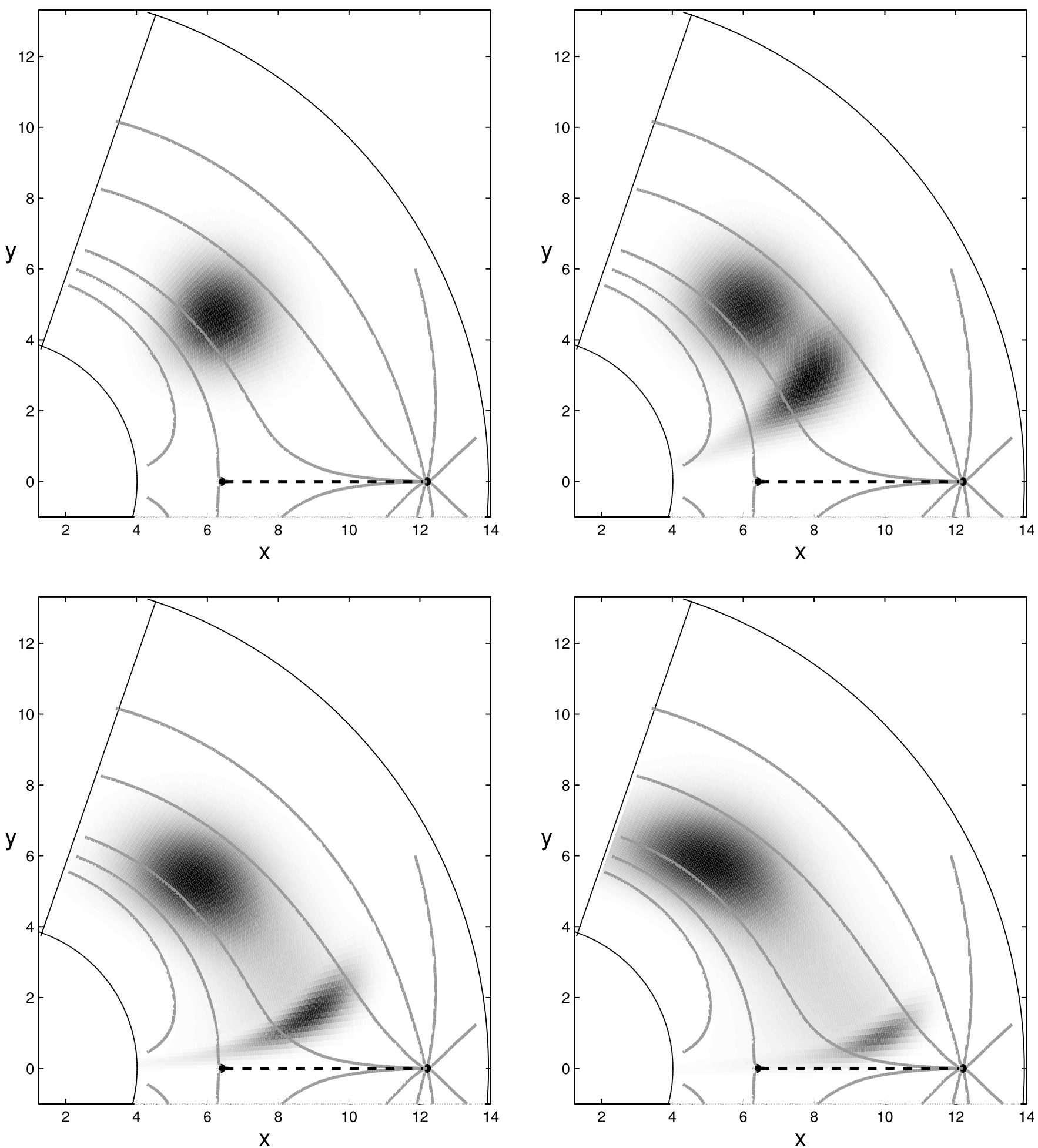

FIG. 4. - Symbols are the same as Fig. 2. The ingoing peak forms a current in the vicinity of the separator. The shading in each image, taken at times $t=0,4.8,11.2$,

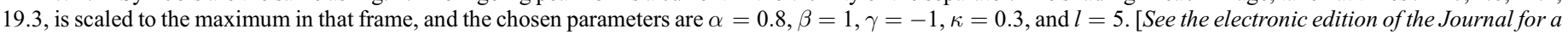
color version of this figure.]

the directional derivative, so that $\mathcal{L} f \rightarrow f_{s}$, then equation (23) becomes

$$
f_{t}+\alpha^{+} f_{s}+\alpha^{-} \frac{\kappa}{l} f=0
$$

with solution

$$
f=f_{0}\left(s e^{\alpha^{+} t}\right) e^{-\alpha^{-}(\kappa / l) t} .
$$

Equation (24) demonstrates the spatial localization of $f$ along the background field lines, for $\alpha>0$ corresponding to inflow in the $r-\theta$ plane, as well as indicating an exponential decay in time. Therefore, for large time we may neglect $f$ in the evolution equation for $b$. Setting $f=0$ in equation (22), we deduce, as above, that

$$
b=b_{0}\left(s e^{\alpha^{-} t}\right) e^{\alpha^{-}(\kappa / l) t},
$$




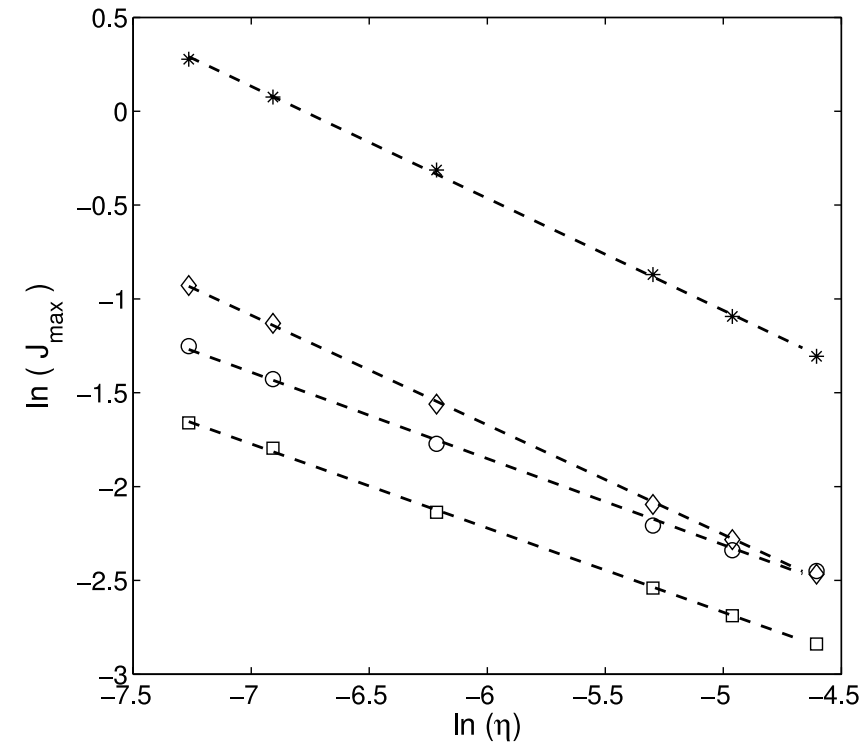

FIG. 5.- Scaling of the peak current with resistivity and best-fit lines to the data, in the regime $|\beta|>|\alpha|$, for the single null with curved fan current buildup (stars), and the double null fan current (diamonds), spine current (squares), and separator current (circles) runs.

which shows a slower localization of $b$ than $f$, coupled with an exponential growth of $b$.

We conclude that the immediate effect of the magnetic perturbation is to drive the velocity disturbance field toward its quasisteady state distribution $[v \approx(\beta / \alpha) b]$. Once the equalization phase has occurred, the disturbance fields begin a gradual localization phase, which is accompanied by exponential growth. This growth is arrested once length scales are sufficiently reduced that nonideal effects become important.

\subsection{Simulation Results}

We now summarize numerical simulations performed in the regime $|\alpha|>|\beta|$. Once again, the initial conditions are chosen such that $v=0$ at $t=0$, and $b$ is some nonzero disturbance pulse. The early phase of the evolution confirms that the velocity profile $v$ does indeed quickly grow [to $v \approx(\beta / \alpha) b$ ], in order to mirror the magnetic disturbance field profile. Once this has happened, the behavior of $v$ closely follows that of $b$, with just a small phase delay. Both disturbance fields are then advected toward the null point pair, with the magnitude of the magnetic field disturbance, as well as the current, growing as the localization proceeds. As described in $\S 4$, the nature of this localization depends on the relationship between the initial disturbance and the background field $\boldsymbol{P}$, and again localization toward the spine, fan, or separator is possible in the double null field. In each case, we require $\alpha>0$ to ensure inflow of the disturbances.

\subsubsection{Fan Reconnection}

We examine first the case of fan reconnection. Consider first an isolated linear three-dimensional background null of the form $\boldsymbol{Q}=\left[\lambda_{1} x,\left(\lambda_{2}-\lambda_{1}\right) y,-\lambda_{2} z\right]$, where $\lambda_{2}>\lambda_{1}>0$. In this case, the fan is the plane $z=0$, and a typical disturbance has $x$ - and $y$-components, which are expected to scale with resistivity as

$$
J_{\max } \sim \eta^{-\mu_{J}}, \quad B_{\max } \sim \eta^{-\mu_{b}}
$$

where $\mu_{J}=(1+A) / 2, \quad \mu_{b}=A / 2$, and $A=\lambda_{1} / \lambda_{2}$ (for the $x$-component) or $\left(\lambda_{2}-\lambda_{1}\right) / \lambda_{2}(y$-component; see Craig \& Fabling 1998; Heerikhuisen \& Craig 2004). Hence, when the null point is isotropic $\left(\lambda_{1}=\lambda_{2} / 2\right)$, each component is equally magnified $(A=1 / 2)$. However, if the null is nonisotropic, and therefore the outflow in the fan is stronger in one direction (as defined by the eigensystem of the null-see $\S 2$ ), then one component will be stretched and magnified more strongly than the other.

Our present purpose is to test whether the above scalings persist in more complicated field configurations for both the single and double null background fields. The disturbance field in our case is always aligned to the $z$-axis, and it is the background fan field that governs the strength of the outflow in this direction. Now, in order to determine the expected scalings (as predicted by the theory for a single linear null point), it is necessary to calculate the appropriate ratio of the eigenvalues of the matrix $\nabla \boldsymbol{B}$ at the null (see $\S 2$ ). As discussed above, this is the ratio of the

TABLE 1

Scaling Results from Simulations and Predictions based on Previous Analytical Work

\begin{tabular}{|c|c|c|c|c|c|c|}
\hline \multicolumn{3}{|c|}{ Run INFO } & \multicolumn{2}{|c|}{$\mu_{J}$} & \multicolumn{2}{|c|}{$\mu_{b}$} \\
\hline Nulls & Localization & $A$ & Observed & Expected & Observed & Expected \\
\hline \multicolumn{7}{|c|}{$|\beta|>|\alpha|$} \\
\hline $1 \ldots \ldots \ldots \ldots \ldots \ldots$ & $\mathrm{F}$ & 0.26 & $0.61 \pm 0.02$ & 0.5 & $\ldots$ & $\ldots$ \\
\hline 1 & $\mathrm{~F}$ & 0.36 & $0.60 \pm 0.02$ & 0.5 & $\ldots$ & $\ldots$ \\
\hline 1 & $\mathrm{~F}$ & 0.45 & $0.58 \pm 0.01$ & 0.5 & $\ldots$ & $\ldots$ \\
\hline 2 & $\mathrm{~F}$ & 0.40 & $0.58 \pm 0.02$ & 0.5 & $\ldots$ & $\ldots$ \\
\hline 2 & $\mathrm{Sp}$ & 0.51 & $0.49 \pm 0.02$ & 0.5 & $\ldots$ & $\ldots$ \\
\hline $2 \ldots \ldots \ldots \ldots \ldots \ldots \ldots \ldots \ldots$ & $\mathrm{Se}$ & $\ldots$ & $0.45 \pm 0.01$ & 0.5 & $\ldots$ & $\ldots$ \\
\hline \multicolumn{7}{|c|}{$|\alpha|>|\beta|$} \\
\hline 1 & $\mathrm{~F}$ & 0.36 & $0.68 \pm 0.03$ & 0.68 & $0.17 \pm 0.03$ & 0.18 \\
\hline 1 & $\mathrm{~F}$ & 0.46 & $0.73 \pm 0.02$ & 0.73 & $0.24 \pm 0.01$ & 0.23 \\
\hline 1 & $\mathrm{~F}$ & 0.70 & $0.87 \pm 0.02$ & 0.85 & $0.37 \pm 0.02$ & 0.35 \\
\hline 2 & $\mathrm{~F}$ & 0.40 & $0.70 \pm 0.02$ & 0.70 & $0.21 \pm 0.02$ & 0.20 \\
\hline 2 & $\mathrm{Sp}$ & 0.54 & $1.50 \pm 0.02$ & 1.43 & $0.98 \pm 0.04$ & 0.93 \\
\hline
\end{tabular}

Notes.-Column (1) gives the number of nulls in the field for that run, column (2) denotes which topological feature the current localizes toward ( $\mathrm{F}$ for fan, $\mathrm{Sp}$ for spine, Se for separator), and $A$ is the isotropy of the corresponding null. Observed results are calculated by linear regression, with errors given at $95 \%$ confidence level. 


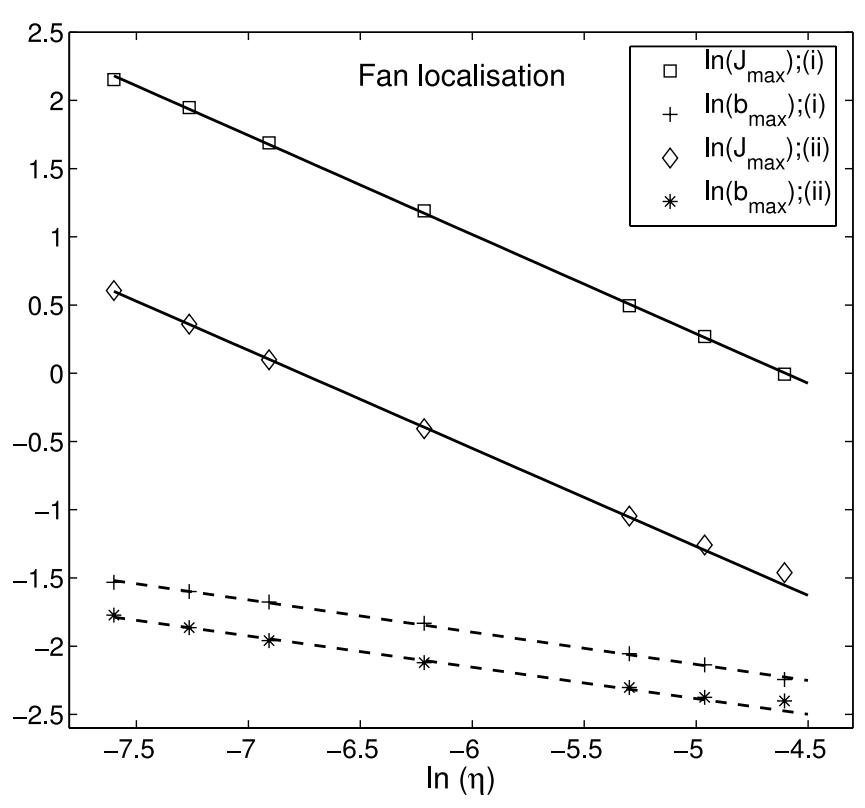

FIG. $6 a$

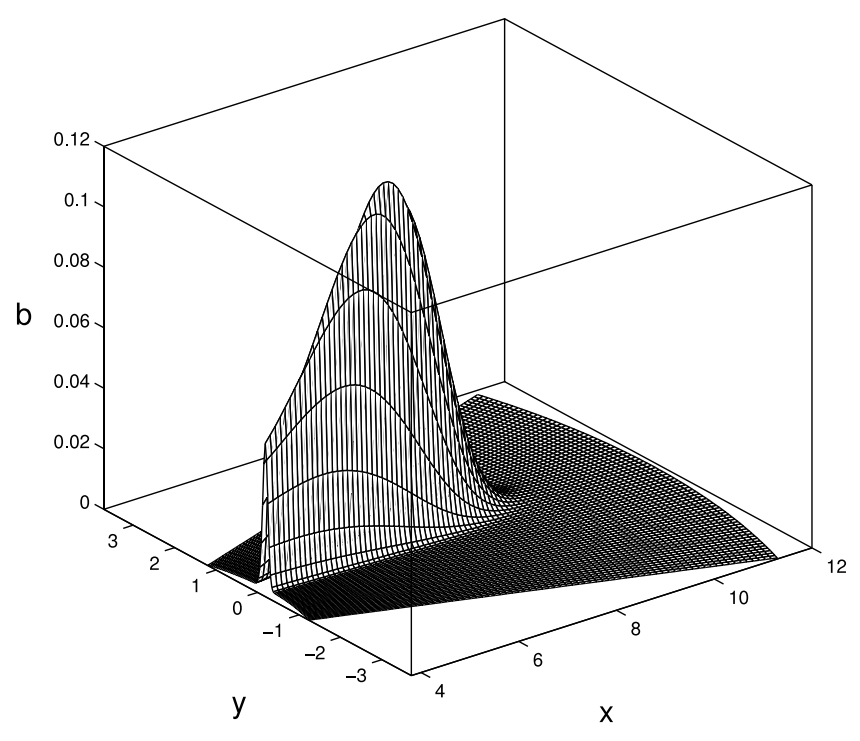

FIG. $6 b$

FIG. 6.- (a) Scaling of the peak current $\left(J_{\max }\right)$ and maximum field at time of peak current $\left(b_{\max }\right)$ with resistivity, and best fit lines, for fan currents in the presence of one null (run i; $\mu_{J}=0.73, \mu_{b}=0.24$ ) and two nulls (run ii; $\mu_{J}=0.70, \mu_{b}=0.21$ ). (b) Typical disturbance field profile at time of $J_{\max }$ for the two-null field, for parameters $\alpha=2, \beta=0.4, \gamma=-1, \kappa=0.33, l=5$.

outflow eigenvalue corresponding to the direction parallel to the disturbance component to the inflow eigenvalue.

For the case of the single null background field, a curved current sheet is formed in the fan plane of the null (the $z-\theta$ plane). The peak current and maximum field at time of peak current are plotted in Figure $6 a$. By comparison with the field $Q$, we see that the parameter $A$ is given in our case by $P_{3 z} / P_{1 r}$. The observed scalings for $J_{\max }$ and $b_{\max }$ for two representative runs, along with the expected values of $\mu_{J}$ and $\mu_{b}$, are given in Table 1 . It can be seen that the results show a very good agreement with the predictions for the much simpler field configuration.

A fan current may also be obtained in the case of the double null background field. Using the same background field parameters and perturbation as those employed in the run shown in Figure 2, the fan localization shown in Figure $6 b$ is obtained. In this case, the relevant degree of stretching due to the exhaust flow is computed via $A=r P_{3 z} / P_{2 \theta}$. Observed (plotted in Fig. $6 a$ ) and expected scalings are given in Table 1 , and they again demonstrate very good agreement between the expected values in simple configurations and the behavior in this more complicated topology.

\subsubsection{Spine Reconnection}

For a general disturbance in the fan plane of null 2, as before, a spine-type current sheet results. A typical localized disturbance is shown in Figure $7 b$, and it should be noted that due to the relatively weak driving flow in the fan close to null 2 , this localization occurs over many Alfvén times.

The single peak (in $b$ ) disturbance corresponds to a solitary rotational "tube" of current centered on the spine. This is the generic current structure for a single disturbance, and a building block for the standard steady state spine solution of Craig \& Fabling (1996), in which, due to the symmetries of the coordinate system employed, the spine current sheet is made up of two such tubes.

The expected scalings in the dynamic regime of spine current sheets are given by $\mu_{J} \approx(1+A) /(2 A)$ and $\mu_{b} \approx 1 /(2 A)$ (for a field of the form $\boldsymbol{Q}$, see Heerikhuisen \& Craig 2004). In this case there are two inflow directions and one outflow $\left(0<\lambda_{1}<\lambda_{2}\right)$, and the fastest scaling is obtained when the inflow speeds are equal $\left(\lambda_{1}=\lambda_{2} / 2, A=1 / 2\right)$, giving cylindrical current structures. When $\lambda_{1} \neq \lambda_{2} / 2$, the cylinder "flattens out," and the scaling weakens. We must therefore take $A$ to be the smaller of $\lambda_{1} / \lambda_{2}$ and $\left(\lambda_{2}-\lambda_{1}\right) / \lambda_{2}$ (for the field $\boldsymbol{Q}$ ), or in our case, the smaller of $P_{3 z} / P_{1 r}$ and $r P_{3 z} / P_{2 \theta}$.

A typical set of results obtained from our simulations is plotted in Figure $7 a$. The resultant scaling parameters are again displayed in Table 1. Although the actual values of $\mu_{J}$ and $\mu_{b}$ are slightly higher than the predicted ones, the basic scaling is of the same order and still "superfast," in the sense that the reconnection rate $\sim \eta J$ will scale as a negative power of $\eta$.

\subsubsection{Separator Reconnection}

One might expect that, as in the $|\beta|>|\alpha|$ regime, a suitably chosen initial disturbance might localize along the separator, to a separator current sheet. This is indeed the case, although one must take care when analyzing such a situation. As before, a pulse initiated in the fan of null 2, but close to the spine of null 1 , will localize toward the separator. However, due to the strong driving flow, the localization and current growth does not halt at this time. Rather, since there is a strong flow along the separator, the disturbance will continue to localize along this direction $(x=z=0)$ and, assuming that the resistivity is sufficiently small, the current will continue to slowly grow until the pulse fully localizes at null 2 , in a spine current sheet. This effect is shown in Figure 8, at an intermediate time in the slow localization along the separator.

In the present simulations, we are limited by all of the usual constraints of numerical resolution. However, one might expect that if the separator is sufficiently long, or equivalently if the disturbance is initiated sufficiently close to the separator, that a diffusive current sheet will form at some location on the separator. For this to occur, we require that the time taken for the disturbance to be advected onto the separator (in the perpendicular 

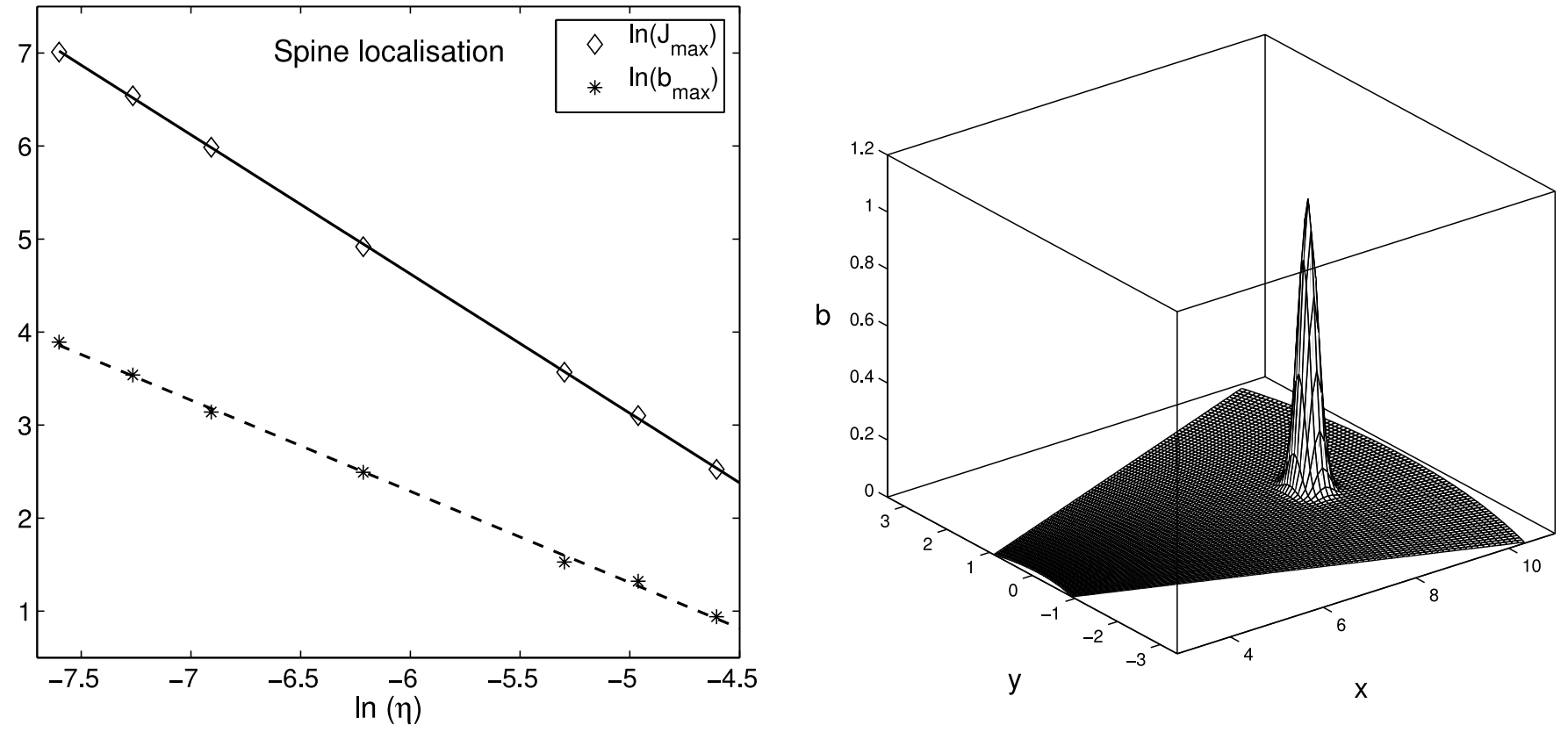

FIG. 7.-Left: Scaling of the peak current $\left(J_{\max }\right)$ and maximum field at time of peak current $\left(b_{\max }\right)$ with resistivity, and best-fit lines, for the spine current, giving $\mu_{J}=1.496, \mu_{b}=0.981$. Right: Typical disturbance field profile at time of $J_{\max }$. Parameters are $\alpha=20, \beta=4, \gamma=-1, \kappa=0.25, l=3$.

direction) and form a sheet $\left(\tau_{\text {rec }}\right)$ be less than the time taken for the flow to push the pulse along the length of the separator $\left(\tau_{\text {sep }}\right)$ to instead form a current sheet at the null point. In order to obtain some estimate for the length scales involved, we approximate our "long" separator (in Cartesian coordinates) by the familiar $\boldsymbol{B}_{s}=\left(x / l, y / l, b_{0}\right)$, where $l$ is a magnetic field length scale across the separator and $b_{0}$ is constant. It can then be shown (e.g., Heerikhuisen \& Craig 2004) that if the disturbance has length scale $l$, then

$$
\tau_{\text {rec }} \sim \frac{l}{2 \alpha^{-}} \ln \left(\frac{\alpha^{-} l}{\eta}\right)
$$

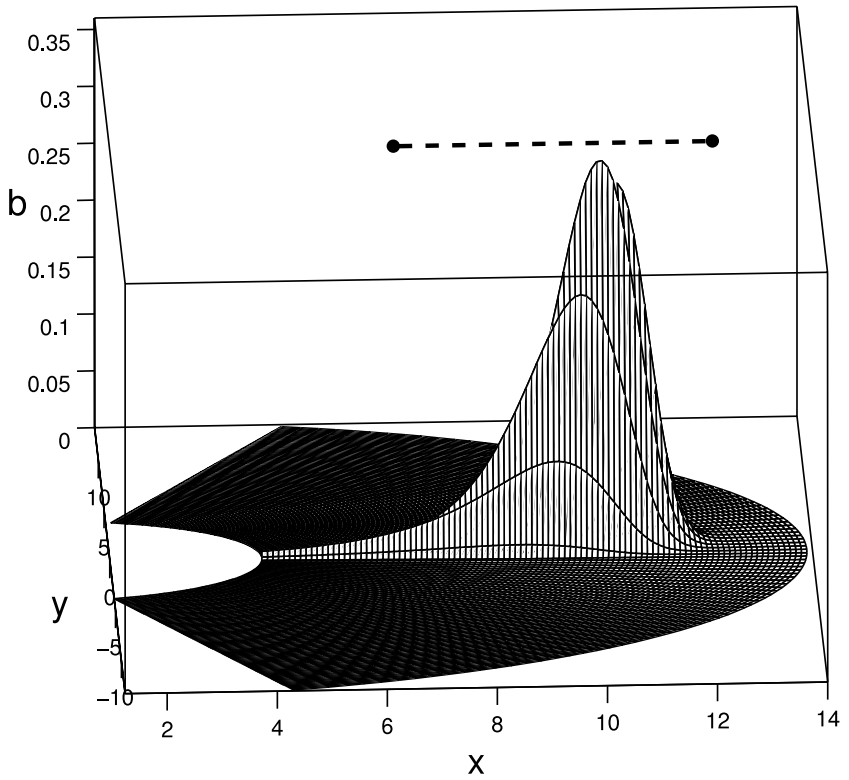

FIG. 8.- Typical disturbance field profile for a separator current, during the slow localization phase along separator, for parameters $\alpha=2, \beta=0.4, \gamma=-1$, $\kappa=0.3, l=5$. Plotted on top of the box are the positions of the null points and separator.
Now, since $\alpha>\beta$, the speed of the pulse along the separator is $\alpha b_{0}$. Thus, if the length of the separator is denoted by $L$, then $\tau_{\text {sep }} \sim L / \alpha b_{0}$. In our system of normalized equations (see $\S 3$ ), we have that $\alpha, \alpha^{-}, b_{0}, L \sim 1$, and therefore in order that $\tau_{\text {rec }} \leq \tau_{\text {sep }}$ we require, if $\eta \sim 10^{-14}$, that $l \lesssim 0.06$. This certainly does not seem out with the realms of possibility in a realistic plasma environment. With $\eta \sim 10^{-3}$, as is typical in our simulations, we require $l \lesssim 0.3$. This, unfortunately, is on the boundary of what we can achieve with numerical resolution, so we have been unable to test this idea properly.

From the above considerations, it seems unlikely that a longlived "separator current sheet" can be formed in this type of flux pileup regime, in which there is always a strong flow along the separator itself. In the present example, the "separator current" buildup has a mixture of fan and spine behaviors, and what is clear is that the nulls themselves play a crucial role in determining this behavior.

Therefore, in order to obtain a quasi-steady state current sheet located along (at least the majority of) the separator, it may be necessary to consider a compressible collapse-type model (e.g., Longcope \& Cowley 1996, or cf. Pontin \& Craig 2005).

\section{SUMMARY}

We have investigated the dynamic behavior of disturbances in the vicinity of a pair of three-dimensional magnetic null points connected by a stable fan-fan separator, in an incompressible, cylindrical geometry. It has been shown that the system can be analyzed in terms of the superposition of transient disturbances onto a background field defining the two nulls. This result generalizes the previous Cartesian description of transient reconnecting disturbances in a three-dimensional single null geometry (Craig \& Fabling 1998), as well as steady state cylindrical merging solutions (Watson \& Craig 2002; Tassi et al. 2003).

The behavior of the system falls into two main regimes, depending on the relative magnitudes of the background magnetic field and plasma flow (here $\beta$ and $\alpha$, respectively). If $|\beta|>|\alpha|$, then the background flow is too weak to localize the disturbance field. In this case the magnetic disturbance develops two peaks, 
and these move in opposite directions along the background field lines. This behavior is unaffected by changing the sign of either $\beta$ or $\alpha$. The pulse that moves inward toward the null point pair provides the reconnecting field that localizes toward either a spine, fan or separator. Of course, a large-scale disturbance, smeared over the entire two-null geometry, may contain regions that are individually focused toward the separator and the nulls. In each case, the localization is accompanied by a current growth, with the peak current scaling at a slow $\eta^{-0.4}$ to $\eta^{-0.6}$ with resistivity. This implies that the resulting reconnection is slow and that the energy release at realistic resistivities is insufficient to account for explosive physical processes such as solar flares. This does not, however, preclude these mechanisms from providing background heating in solar or stellar coronae. Rather, our results provide support for magnetic dissipation theories that propose separatrices and separators as preferred sites for background coronal heating (e.g., Priest et al. 2005), by demonstrating how transient disturbances are channeled by the background field lines toward such topological features.

In order to speed up the reconnection rate, it is necessary to consider the "flux pileup" regime associated with strong driving flows $|\alpha|>|\beta|$. In this regime, both wave solutions for the initial disturbance field travel in the same direction. Initially, there is a relatively fast equalization of the magnetic and velocity disturbances to a quasi-steady state $(v \approx(\beta / \alpha) b)$. Assuming that the sign of $\alpha$ is chosen to provide inflow, disturbances are localized toward the null separatrices and may form spine-aligned current sheets, or fan-aligned current sheets, whose scaling properties with resistivity closely match those at isolated linear nulls in Cartesian geometries. In particular, spine currents scale at a superfast rate $J \simeq \eta^{-1.5}$, whereas fan current sheet reconnection models pro- vide more modest current amplitudes of order $\eta^{-0.5}$ to $\eta^{-1}$, depending on the outflow geometry in the fan.

For certain initial parameters and disturbances, a strong separator current may also result. This current, however, may not be sustained by the flow geometry. Specifically, the strong driving flow tends to push the pulse toward the spine of the null point whose fan plane corresponds to the inflow direction. Whether the current concentration grows sufficiently strong to begin diffusive/ reconnective processes at the separator, or whether this occurs once the disturbance has localized at one of the nulls, depends crucially on the length scales parallel and perpendicular to the separator. Since the connected null point pair we consider is completely generic as far as the basic field structure is concerned, this behavior may well be a general property of two null geometries in strongly driven, incompressible regimes. This interpretation is consistent with the steady state results of (Watson \& Craig 2002 ), who found current on the separator only for very special choices of boundary conditions. Hence, when modeling energetic physical processes occurring via reconnection at current sheets along separators, it may be preferable to consider a compressible collapse-type model for the current sheet formation rather than a strongly driven flux pileup mechanism. Notably, in the dynamic models described here, the separator localization contains a hybrid of fan-type and spine-type behaviors that is strongly affected by the two nulls themselves.

The authors would like to acknowledge financial support from the Marsden Fund, grant 02-UOW-050 MIS, and also useful discussions with Paul Watson.

\section{APPENDIX}

The same reduction procedure as described in $\S 3$ may be performed for the disturbance form $\boldsymbol{b}_{2}=a(r, t) \hat{\boldsymbol{\theta}}+b(r, t) \hat{z}$. The resulting differential equations to be solved are

$$
\begin{gathered}
a_{t}=\left(P_{1 r}+\frac{\kappa}{l}+P_{1} \partial_{r}\right)(\beta u-\alpha a)+\eta\left(a_{r r}+\frac{a_{r}}{r}-\frac{a}{r^{2}}\right) \\
b_{t}=\left(P_{1} \partial_{r}+\frac{\kappa}{l}\right)(\beta v-\alpha b)+\eta\left(b_{r r}+\frac{b_{r}}{r}\right) \\
u_{t}=\frac{\beta}{r}\left[P_{1}(r a)_{r}+\gamma l \int_{r_{\min }}^{r} \frac{(r a)_{r}}{r^{2}} d r\right]-\frac{\alpha}{r}\left[P_{1}(r u)_{r}+\gamma l \int_{r_{\min }}^{r} \frac{(r u)_{r}}{r^{2}} d r\right]+\nu\left(u_{r r}+\frac{u_{r}}{r}-\frac{u}{r^{2}}\right)+g(t) \\
v_{t}=\left(P_{1} \partial_{r}-\frac{\kappa}{l}\right)(\beta b-\alpha v)+\nu\left(v_{r r}+\frac{v_{r}}{r}\right)+h(t) .
\end{gathered}
$$

\section{REFERENCES}

Albright, B. J. 1999, Phys. Plasmas, 6, 4222

Antiochos, S. K., Karpen, J. T., \& DeVore, C. R. 2002, ApJ, 575, 578

Beveridge, C., \& Longcope, D. W. 2005, Sol. Phys., 227, 193

Craig, I. J. D., \& Fabling, R. B. 1996, ApJ, 462, 969 1998, Phys. Plasmas, 5, 635

Craig, I. J. D., Fabling, R. B., Heerikhuisen, J., \& Watson, P. G. 1999, ApJ, 523,838

Craig, I. J. D., Fabling, R. B., Henton, S. M., \& Rickard, G. J. 1995, ApJ, 455, L197

Craig, I. J. D., \& Henton, S. M. 1995, ApJ, 450, 280

Craig, I. J. D., \& Watson, P. G. 2003, Sol. Phys., 214, 131 2005, Phys. Plasmas, 12, 2306

Fletcher, L., Metcalf, T. R., Alexander, D., Brown, D. S., \& Ryder, L. A. 2001, ApJ, 554, 451

Fukao, S., Ugai, M., \& Tsuda, T. 1975, Rep. Ion. Sp. Res. Japan, 29, 133

Heerikhuisen, J., \& Craig, I. J. D. 2004, Sol. Phys., 222, 95

Inverarity, G. W., \& Priest, E. R. 1999, Sol. Phys., 186, 99

Ji, H. S., \& Song, M. T. 2001, ApJ, 556, 1017

Lau, Y. T., \& Finn, J. M. 1990, ApJ, 350, 672
Litvinenko, Y. E., \& Craig, I. J. D. 2003, Sol. Phys., 218, 173

Longcope, D. W. 1996, Sol. Phys., 169, 91

Longcope, D. W., Brown, D. S., \& Priest, E. R. 2003, Phys. Plasmas, 10, 3321 Longcope, D. W., \& Cowley, S. C. 1996, Phys. Plasmas, 3, 2885

Longcope, D. W., Kankelborg, C. C., Nelson, J. L., \& Petsov, A. A. 2001, ApJ, 553,429

Longcope, D. W., McKenzie, D., Cirtain, J., \& Scott, J. 2005, ApJ, 630, 596

Longcope, D. W., \& Noonan, E. J. 2000, ApJ, 542, 1088

Parnell, C. E., \& Galsgaard, K. 2004, A\&A, 428, 595

Parnell, C. E., Smith, J. M., Neukirch, T., \& Priest, E. R. 1996, Phys. Plasmas, 3, 759

Pontin, D. I., \& Craig, I. J. D. 2005, Phys. Plasmas, 12, 72112

Priest, E. R., Longcope, D. W., \& Heyvaerts, J. F. 2005, ApJ, 624, 1057

Priest, E. R., \& Titov, V. S. 1996, Phil. Trans. R. Soc. Lond. A, 354, 2951

Schrijver, C. J., \& Title, A. M. 2002, Sol. Phys., 207, 223

Tassi, E., Titov, V. S., \& Hornig, G. 2003, Phys. Plasmas, 10, 448

Watson, P. G., \& Craig, I. J. D. 2002, Sol. Phys., 207, 337

Wheatland, M. S. 2002, Sol. Phys., 208, 33 